\title{
Fracture of the cone of a morse taper junction in revision arthroplasty of the hip
}

\author{
Alexander Schuh ${ }^{1 *}$, Werner Kachler ${ }^{2}$, Stefan Sesselmann ${ }^{3}$, Wolfgang Hönle ${ }^{1}$ and Tarun Goyal ${ }^{4}$ \\ ${ }^{1}$ Musculoskeletal Center, Neumarkt Hospital, 92318 Neumarkt, Germany \\ ${ }^{2}$ Zentrum für Werkstoffanalytik Lauf GmbH, 91207 Lauf a.d. Pegnitz, Germany \\ ${ }^{3}$ Department of Orthopaedics, University of Erlangen- Nurenberg, Erlangen, Germany \\ ${ }^{4}$ Department of Orthopaedics, All India Institute of Medical Sciences, Rishikesh, India
}

\begin{abstract}
Since the mid-1980s, there has been a dramatic increase in the use of modular THA designs. Surgeons adopted head-neck modularity for the intraoperative advantages of adjusting leg length and femoral offset and neck-stem modularity for the opportunity to further fine-tune hip biomechanics and femoral anteversion. Fretting, crevice and galvanic corrosion, component loosening, and mismatch have all been associated with the use of modular prostheses in primary and revision hip arthroplasty. Dissociation and fracture of modular femoral neck prosthesis are rare and underestimated complications in modular THA. We report the first case of a fracture of the cone of the morse taper junction of a MRP Titan revision stem.
\end{abstract}

\section{Introduction}

Since the mid-1980s, there has been a dramatic increase in the use of modular THA designs. Recently, dual modular stems have been introduced that include an additional neck-stem taper junction. Surgeons adopted head-neck modularity for the intraoperative advantages of adjusting leg length and femoral offset and neck-stem modularity for the opportunity to further fine-tune hip biomechanics and femoral anteversion. Modularity may also provide intraoperative flexibility when partial hip implant revision is required [1-3]. However, increasing modularity also renders several postulated disadvantages that must be taken into consideration.

Fretting, crevice and galvanic corrosion, component loosening, and mismatch have all been associated with the use of modular prostheses in primary and revision hip arthroplasty [4-8]. Dissociation and fracture of modular femoral neck prosthesis are rare and underestimated complications in modular THA [2,4,10-15]. In several publications the MRP Titan revision stem has proven to be a reliable implant in revision surgery of the hip joint $[2,3,16]$. To our knowledge we report the first case of a fracture of the cone of the morse taper junction of a MRP Titan revision stem 3 years after index operation of a 49 years old male patient.

\section{Case Report}

In August 2013 THA was performed due to a girdlestone situation after septic osteoarthritis of the hip (Figure 1) in a 46 year old male patient using a MRP Titan revision stem (Diameter: 18mm, length: 140 $\mathrm{mm}$ ) (Peter Brehm GmbH, Weisendorf, Germany) and a cementless cup (Allofit - IT; Diameter $54 \mathrm{~mm}$, Zimmer, Warsaw, USA) and a ceramic on ceramic bearing (head diameter: $36 \mathrm{~mm}$ ) (Figure 2). The patient was $175 \mathrm{~cm}$ tall and weighed $105 \mathrm{~kg}$ (body mass index: $34.3 \mathrm{Kg}$ / $\mathrm{m}^{2}$ ). 2 weeks later revision surgery due to recurrent dislocation (Figure 3) was performed. Revision of the acetabular component was done using an Allofit - Classic- cup, Diameter $56 \mathrm{~mm}$ (Zimmer, Warsaw, USA)). The neck component was exchanged from small to medium size
(Figure 4). A ceramic on PE bearing was implanted (head diameter: 32 $\mathrm{mm}$ ). Postoperative healing was uneventful. The patient was out of any complaints. In February 2016 the patient started suffering from slight thigh pain but detected crepitation in the region of the left hip. X-ray of the left hip in 2 planes in May 2016 revealed an asymmetrically gap of the stem / neck junction (Figure 5). During revision surgery of the left hip breakage of the cone of the morse taper junction was visible (Figure 6). Removal of the well-fixed stem was performed via a distal fenestration. After fixation with cerclage wires a bended MRP Titan revision stem (Diameter: $19 \mathrm{~mm}$, Length: $200 \mathrm{~mm}$ with long neck and extension sleeve) was implanted using a $32 \mathrm{~mm}$ ceramic head (Figure 7). Postoperative healing was uneventful.

Light microscopy and field emission scanning electron microscopy (SEM, LEO 1525) was used for analyzing the broken cone. The fracture of the male cone showed typical secondary damage features attributed to handling, especially manipulation during explanation procedure. These never avoidable deteriorations hindered the possibility of analyzing the very starting point of the fracture. Additionally, hinderness was the fact that the overweight patient walked about 3 months with the broken cone. Globally the fracture is of a typical fatigue type, starting surprisingly deep inside the cone contact surfaces. To reveal specific features of the contact area at the crack initiation site we opened the distal part of the component exact axially in order to prepare carefully out the male cone residue. Light microscopy and

Correspondence to: Prof. Dr. Alexander Schuh, Head of Musculoskeletal Center, Neumarkt Hospital, Teaching Hospital of the University of Erlangen-Nurenberg, Nürnberger Str. 1292318 Neumarkt i.d. Opf, Germany, Tel: +49-9181-420-3500; Fax: +49-9181-420-3503; E-mail: Alexander.Schuh@klinikum.neumarkt.de

Key words: MRP titan revision stem, fracture, cone, morse taper junction, modular

Received: March 14, 2018; Accepted: March 28, 2018; Published: April 02, 2018 


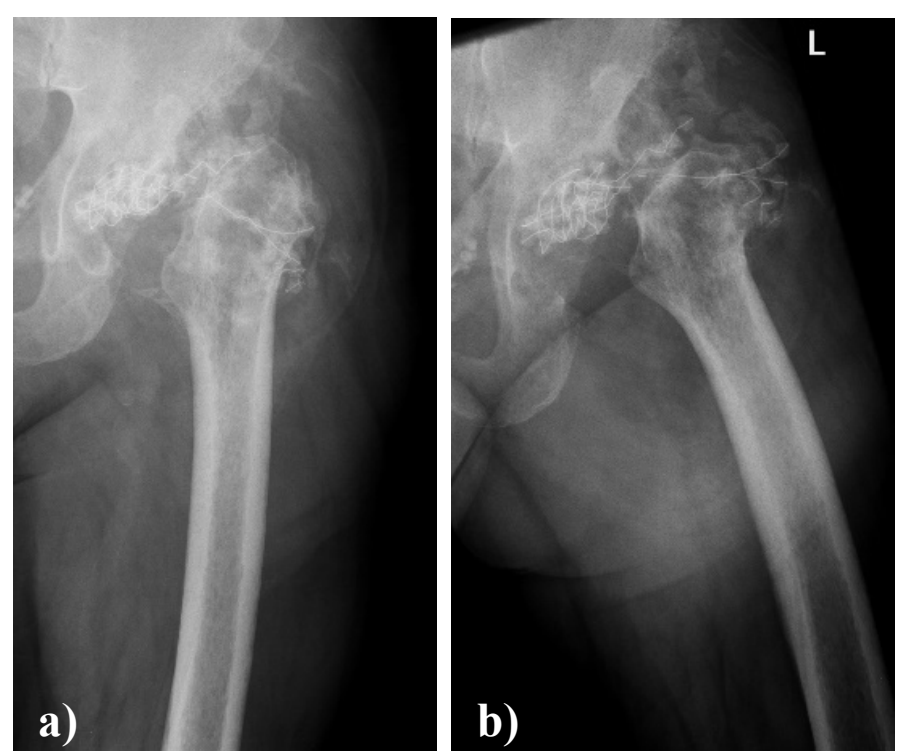

Figure 1. X-ray of the left hip in 2 planes: ap view a. and lateral; b. view shows a girdlestone situation following septic arthritis of the left hip with staph. aureus.

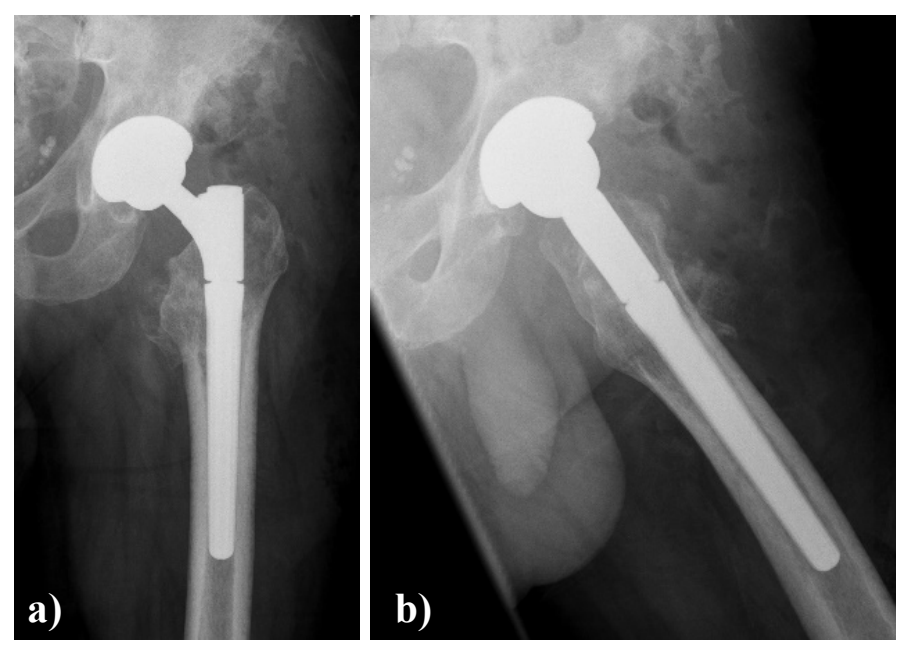

Figure 2. $X$ - ray of the left hip in 2 planes: ap view a. and lateral; b. view shows THA using a MRP Titan revision stem with a short neck and a cementless cup.

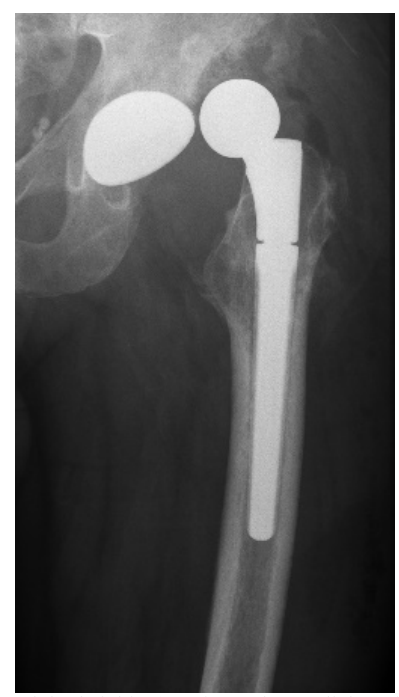

Figure 3. X - ray of the left hip (ap view) shows dislocation of the left hip.
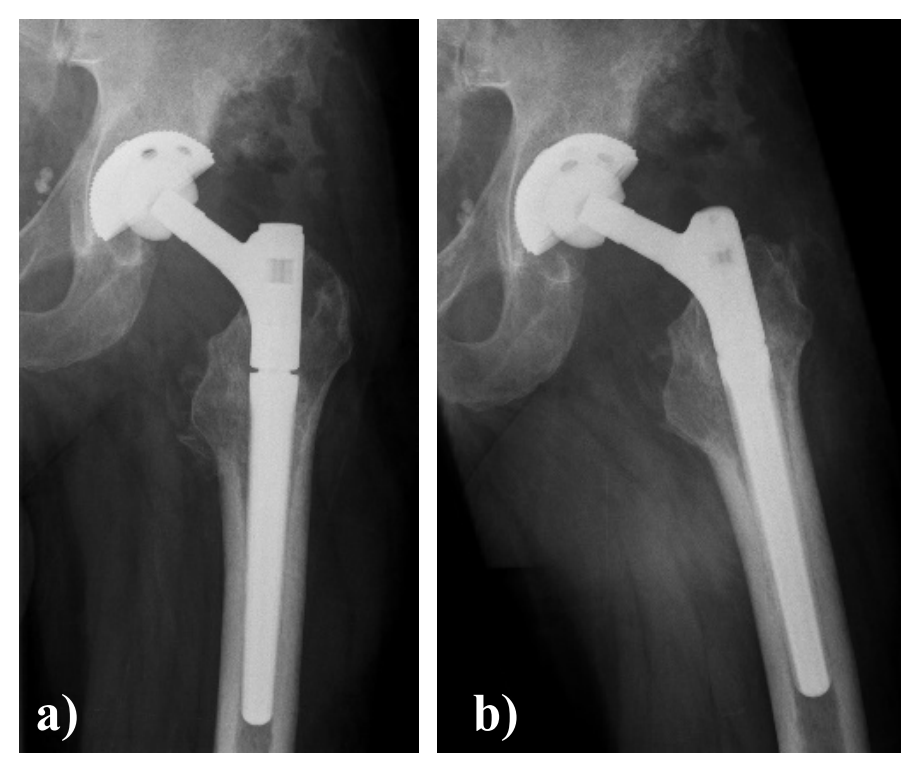

Figure 4. $X$ - ray of the left hip in 2 planes: ap view a. and lateral; $b$ view shows status afte revision of THA using a long varus neck and a cementless cup.
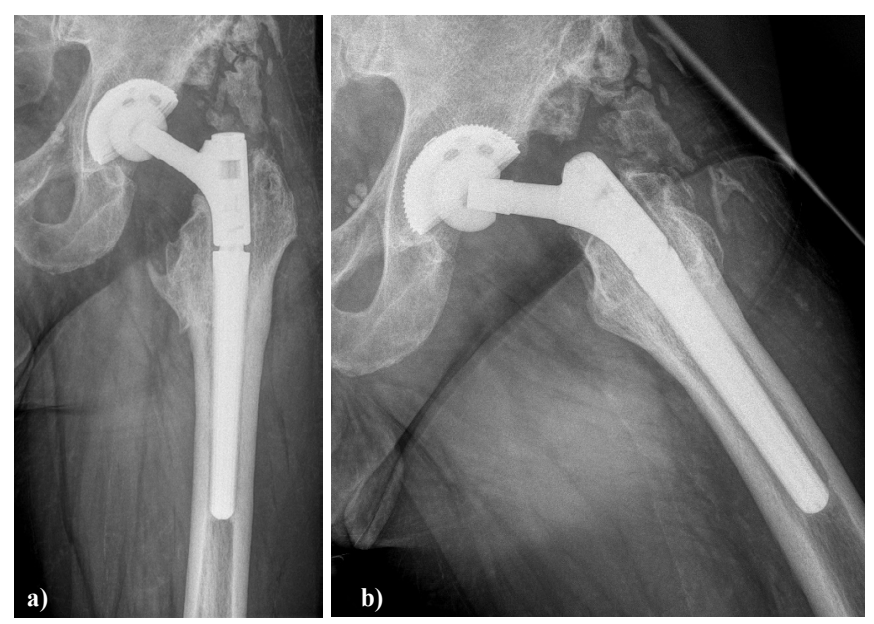

Figure 5. $X$ - ray of the left hip in 2 planes: ap view a. and lateral; b. of the left hip reveals an asymmetrically gap of the stem / neck junction and radiolucent lines in zone 1,7,8, and 14 according to Gruen.
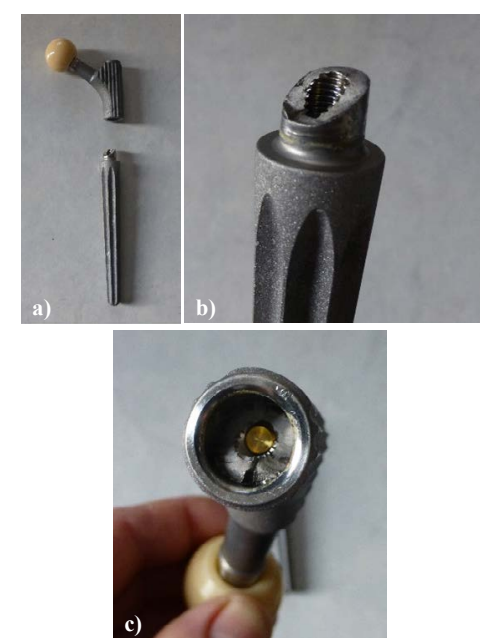

Figure 6. $\mathrm{a}$ and $\mathrm{b}$ showing the broken cone of the morse taper junction; $\mathrm{c}$. view into the female part of the morse taper junction showing the broken cone and broken golden expansion screw. 


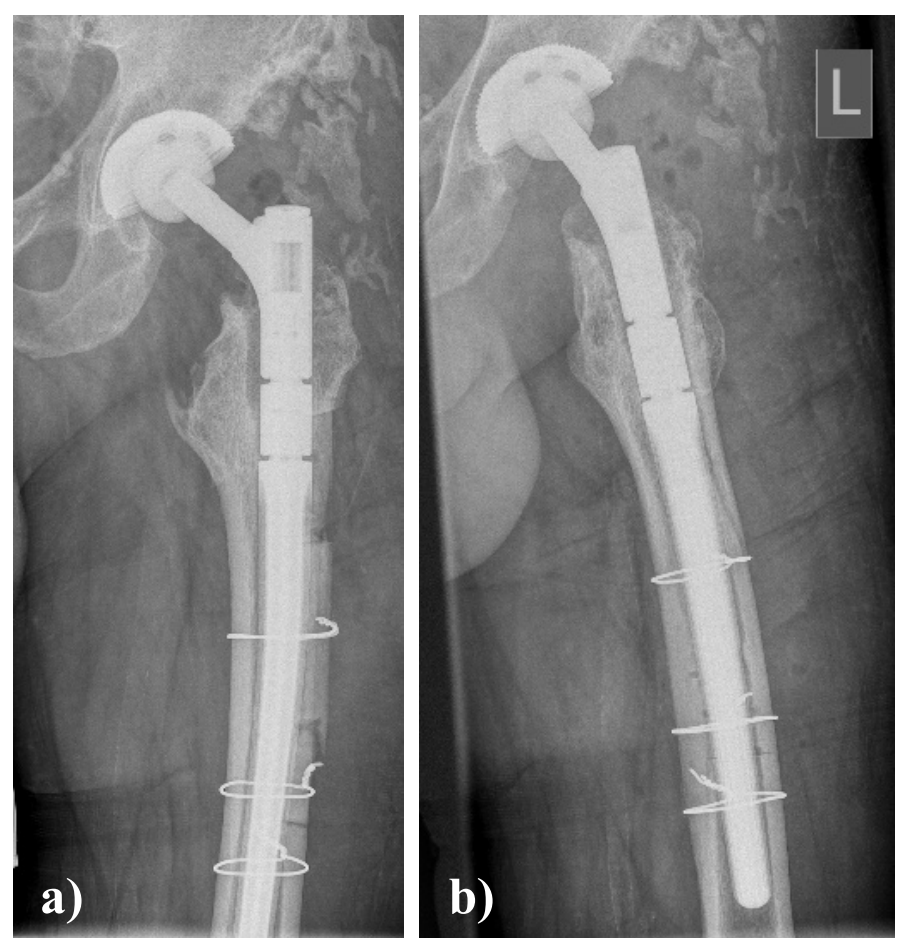

Figure 7. $\mathrm{X}$ - ray of the left hip in 2 planes: ap view a. and lateral; b. view shows revision arthroplasty of the hip using a long bended MRP Titan revision stem with long neck and extension sleeve.

field emission scanning electron microscopy revealed fretting and crevice corrosion and fatigue crack (Figure 8-10). There were no signs of design or material flaws.

\section{Discussion}

Modular THAs incorporating a double-taper (neck-head and neck-stem) design are a recent innovation in hip arthroplasty and have distinct advantages compared to single neck-head taper or monolithic designs. Intraoperative flexibility is clearly increased by using a modular-neck device. Clinical advantages of modular necks include the adjustment of leg length and offset via the head-neck taper, femoral anteversion via the neck-stem taper, easier revision when there is no clinical need to revise a well-fixed stem, and optimal restoration of soft tissue tension and patient biomechanics [1-3]. Despite the recent popularity of increasing modularity, there is a paucity of studies that have been published reporting modular femoral neck failure $[4,13$ 15,17]. In the reported case of Dangles et al. [9] -who reported the first modular femoral neck fracture in an obese, active man - potential design flaws were discussed for implant failure. To date, most reported fatigue fractures of modular stems have been associated with titanium (Ti6Al4V) alloy neck and stem components [4]. Dunbar et al. [18] postulated an implant neck fracture rate of $1.4 \%$ in Ti modular-neck prostheses. Skendzel et al. [13] presented 2 cases of modular prosthesis fracture. They discussed that the combination of patient obesity with a long modular neck may have contributed to modular neck fracture. Specifically, the use of a long varus neck increases the bending moment by $32.7 \%$ compared with the standard "short varus" neck, with increasing stress concentration at the modular junction $[4,13]$. There are examples of fracture in the modular neck of modern hip implants. Most prominently reported is the Profemur-Z (Wright Medical Technology, Arlington, Tennessee), which in the Australian market has shown an unacceptably high failure rate of $11.2 \%$ at the modular stem-body tapered junction [19]. On a microscopic level, micromotion at the junctional interface induces both fretting and crevice corrosion, theoretically contributing to the creation of microcracks within the zone of corrosion and increasing the risk of dynamic fatigue fracture. Both titanium and cobalt chrome alloys form a protective oxide layer that aids in resisting corrosion, termed self-passivation. With increased stresses on the implants, particularly at modular head/neck and neck/ stem junctions, these oxide layers are disrupted, leading to a vicious cycle with recurrent attempts of the metal to repassivate their surface, subsequently depleting the available oxygen supply, releasing chloride ions within the joint and lowering the local $\mathrm{pH}$. This creates an anaerobic and acidic environment more susceptible to further abrasive wear and corrosion, potentiating the risk of component fracture over time [4]. Ti-based devices have higher corrosion breakdown potentials than CoCr-Mo devices [1]. The combination of corrosion, large femoral head components with long modular necks, metal-on-metal components, patient obesity, and activity level may create a local microenvironment that can initiate and perpetuate fatigue failure. In the case of Ellmann [4] the combination of these factors led to the "perfect storm" for fretting,

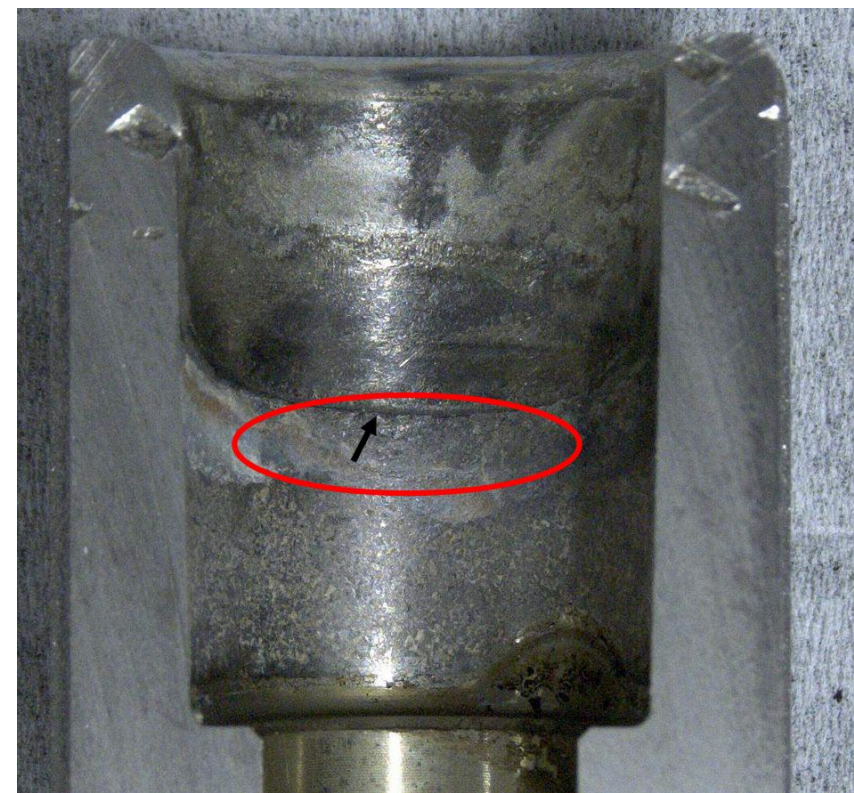

Figure 8. Insight of the female component: Border line of the crack path. Below the crack initiation site the contact cone surfaces show massive fretting and fretting oxidation $(\mathrm{O})$.

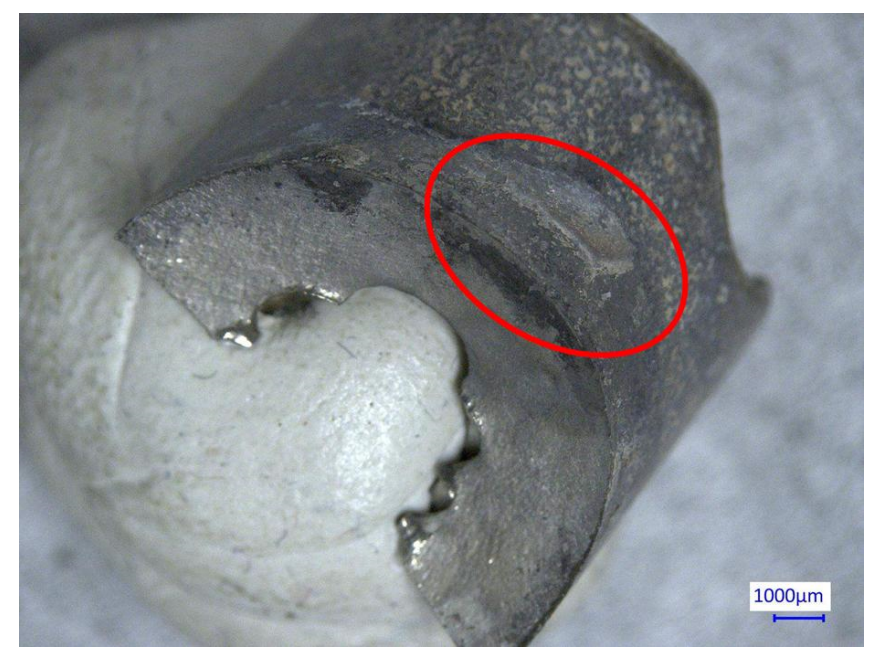

Figure 9. At the male cone the fretting features are also clearly visible $(\mathbf{O})$. The surface below the contact fretting pattern additionally shows features of crevice corrosion. 


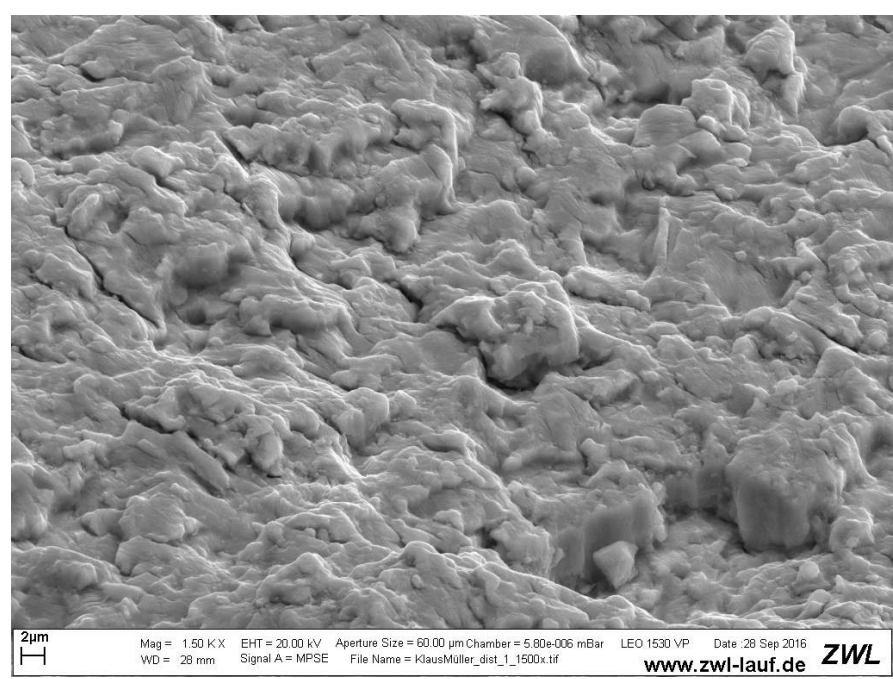

Figure 10. The crack surface itself reveals striations with very narrow distance, typical of a fatigue crack propagation with low velocity over a long period.

corrosion, and failure. Increased stress over a long lever arm creates an environment susceptible to modular component fracture/failure. Kop et al. [1] found the severity of corrosion was greater at the neck-stem junction than at the head-neck junction. This is not unexpected as the forces at the head-neck junction are transferred through a spherical bearing resulting in relatively low contact stresses, whereas there is eccentric loading at the neck-stem junction and depending on the offset and length of the neck, these stresses can be very high. A recent study of S-ROM1 THAs revealed that $27 \%$ of retrieved devices could not be disassociated in theatre due to cold welding. This may prevent the production of metallic debris, but a key design feature of these devices -partial replacement in revision surgery- failed [1]. Lakstein et al. [20] analysed six patients with a fracture at the mid-stem junction of a modular revision hip implant They concluded that the stem failure was initiated by a fretting fatigue mechanism and was propagated by a pure bending fatigue mechanism. Risk factors for fractures of the modular junction include excessive body weight and inadequate proximal osseous support because of trochanteric osteotomy, reduced preoperative bone stock, osteolysis, loosening, and/or implant under sizing. Surgeons should consider the use of implants with strengthened junctions when using modular stems in such patients. A lot of research has been done to minimize corrosion using shot peening procedures $[2,3]$ or deep rolling [16] to induce compressive residual stresses to the male part of the morse taper junction. The contamination of the morse taper junction with body liquid can enhance corrosion in the junction gap as well as fatigue through stress corrosion cracking [16]. In our case there were no signs of design or material flaws of the cone or surgical flaws. Maybe the fracture of the cone was a product of the "perfect storm" according to Ellmann et al. [4].

\section{Conclusion}

We follow Ellmann et al. [4] that with increasing use of modularity, fracture of the modular neck is a real but rare and likely underreported concern in THA. Surgeons must be aware of its potential complications and plan accordingly. Long varus modular necks in obese patients seem to be important risk factors for neck fracture in modular femoral stems.

\section{Compliance with ethical standards}

Conflict of Interest: The authors declare that they have no conflict of interest.
Funding: There is no funding source.

Ethical approval: This article does not contain any studies with human participants or animals performed by any of the authors.

\section{References}

1. Kop AM, Keogh C, Swarts E (2012) Proximal component modularity in THA--at what cost? An implant retrieval study. Clin Orthop Relat Res 470: 1885-1894. [Crossref]

2. Schramm M, Wirtz DC, Holzwarth U, Pitto RP (2000) The Morse taper junction in modular revision hip replacement--a biomechanical and retrieval analysis. Biomed Tech (Berl) 45: 105-119. [Crossref]

3. Schuh A, Werber S, Holzwarth U, Zeiler G (2004) Cementless modular hip revision arthroplasty using the MRP Titan Revision Stem: outcome of 79 hips after an average of 4 years' follow-up. Arch Orthop Trauma Surg 124: 306-309. [Crossref]

4. Ellman MB, Levine BR (2013) Fracture of the modular femoral neck component in total hip arthroplasty. J Arthroplasty 28: 196.e1-5. [Crossref]

5. Esposito CI, Wright TM, Goodman SB, Berry DJ (2014) What is the trouble with trunnions? Clin Orthop Relat Res 472: 3652-3658. [Crossref]

6. Munir S, Walter WL, Walsh WR (2015) Variations in the trunnion surface topography between different commercially available hip replacement stems. J Orthop Res 33: 98105. [Crossref]

7. Pour AE, Borden R, Murayama T, Groll-Brown M, Blaha JD (2016) High Risk of Failure With Bimodular Femoral Components in THA. Clin Orthop Relat Res 474: 146-153. [Crossref]

8. Wassef AJ, Schmalzried TP (2013) Femoral taperosis: an accident waiting to happen? Bone Joint J 95-B: 3-6. [Crossref]

9. Dangles CJ1, Altstetter CJ (2010) Failure of the modular neck in a total hip arthroplasty. J Arthroplasty 25: 1169. [Crossref]

10. Fanuele J, Bernini P (2007) Dissociation of the modular femoral stem from the metaphyseal sleeve during reduction of a total hip arthroplasty dislocation . J Arthroplasty 22: 140-142. [Crossref]

11. Higgs GB1, Hanzlik JA, MacDonald DW, Gilbert JL, Rimnac CM, et al. (2013) Is increased modularity associated with increased fretting and corrosion damage in metal-on-metal total hip arthroplasty devices?: a retrieval study. J Arthroplasty 28: 2-6. [Crossref]

12. Kouzelis A1, Georgiou CS, Megas P (2012) Dissociation of modular total hip arthroplasty at the neck-stem interface without dislocation. J Orthop Traumatol 13 221- 224. [Crossref]

13. Skendzel JG1, Blaha JD, Urquhart AG (2011) Total hip arthroplasty modular neck failure. J Arthroplasty 26: 338. [Crossref]

14. Wilson DA, Dunbar MJ, Amirault JD, Farhat Z (2010) Early failure of a modula femoral neck total hip arthroplasty component: a case report. J Bone Joint Surg Am 92: 1514-1517. [Crossref]

15. Wright G1, Sporer S, Urban R, Jacobs J (2010) Fracture of a modular femoral neck after total hip arthroplasty: a case report. J Bone Joint Surg Am 92: 1518-1521. [Crossref]

16. Schuh A, Zeller C, Holzwarth U, Kachler W, Wilcke G, et al. (2007) Deep rolling of titanium rods for application in modular total hip arthroplasty. J Biomed Mater Res B Appl Biomater 81: 330-335. [Crossref]

17. Atwood SA, Patten EW, Bozic KJ, Pruitt LA, Ries MD (2010) Corrosion- induced fracture of a double-modular hip prosthesis: a case report. J Bone Joint Surg (Am) 92 1522-1525. [Crossref]

18. Dunbar MJ (2010) The proximal modular neck in THA: a bridge too far: affirms Orthopedics 33: 640. [Crossref]

19. Parisi T, Burroughs B, Kwon YM (2015) Modular hip implant fracture at the stemsleeve interface. Orthopedics 38: e234-9. [Crossref]

20. Lakstein D, Eliaz N, Levi O, Backstein D, Kosashvili Y, et al. (2011) Fracture of cementless femoral stems at the mid-stem junction in modular revision hip arthroplasty systems. J Bone Joint Surg (Am) 93 : 57-65. [Crossref]

Copyright: (C2018 Schuh A. This is an open-access article distributed under the terms of the Creative Commons Attribution License, which permits unrestricted use, distribution, and reproduction in any medium, provided the original author and source are credited. 